\title{
WEB Geographic Information System and the Hardware and Software Ensuring Rapid Assessment of Air Pollution
}

\author{
O. E. Yakubailik ${ }^{a, b, *}$, A. A. Kadochnikov ${ }^{a}$, and A. V. Tokarev ${ }^{a}$ \\ ${ }^{a}$ Institute of Computational Modeling, Siberian Branch, Russian Academy of Sciences, \\ Akademgorodok 50, building 44, Krasnoyarsk, 660036 Russia \\ ${ }^{b}$ Siberian Federal University, \\ pr. Svobodnyi 79, Krasnoyarsk, 660041 Russia \\ *E-mail: oleg@icm.krasn.ru
}

Received January 16, 2018

\begin{abstract}
This paper touches upon the experience of research and development of hardware and software for air pollution monitoring of industrial cities, carried out at the Institute of Computational Modeling of the Siberian Branch of the Russian Academy of Sciences. Functional possibilities, architectural features, and user interface of the distributed web geographic information system of ecological monitoring of the city of Krasnoyarsk, which is being developed in service-oriented architecture, are discussed. Data are collected from automated posts for observing the state of atmospheric air and services of aggregation and representation of information in the form of interactive graphs and thematic maps in the web interface. An original device is developed for measuring the pollution level of the atmosphere with fine dust (PM2.5), the data from which is fed in real time to the system via a cellular network. A system is formed for integral assessment of the pollution level of the atmosphere based on Air Quality Index (AQI) calculations.
\end{abstract}

Keywords: web GIS, web services, environmental monitoring, AQI, atmospheric pollution, suspended particles, fine dust, spatial data, geoportal, Arduino, aerosol.

DOI: $10.3103 / \mathrm{S} 8756699018030056$

\section{INTRODUCTION}

Systems for monitoring the atmospheric air pollution are developed and introduced in many cities in Russia and abroad. For example, in the Kaluga region, a system for analyzing and managing the atmospheric air quality operates, and its information support is based on the "Vozdukh-Gorod" software-analytical complex [1]. The "Tion" company formed a network of 16 small stations with sensors continuously measuring the concentration of aerosol particles PM2.5 and PM10, as well as humidity, temperature, and atmospheric pressure. Data from this system are available online [2]. The automated system for monitoring atmospheric air, which is developed in St. Petersburg, regularly provides information on air in the city to the authorities and other professional organizations [3]. The hardware-software system for estimating the atmospheric air pollution, developed in the city of Tula, collects information by various types of sensors, uses different engineering devices, carries out a comparative analysis of the data obtained, and solves the most important problems, such as determination of the contribution of particular facilities to atmospheric air pollution at a current moment [4].

It should be noted that there is a German system for monitoring the atmosphere, based on web interfaces and services, in accordance with the specifications of INSPIRE spatial data infrastructure, developed by the European Union [5]. Romanian researches develop a distributed system for atmospheric pollution monitoring by PM2.5 fine dust with a web interface on the basis of a geographic information system (GIS) [6]. Chinese specialists work on special means for air pollution monitoring, which is a TaskML script language and a Sensor Web system for processing and analyzing data from the atmospheric pollution sensors, based on 
TaskML [7]. Italian developers created a system for monitoring the air quality and traffic streams, in which self-made sensors operate, created on the basis of Arduino, and the results are published in a web portal [8]. An unconditional trend is the introduction of web-based solutions based on a distributed network with respect to cheap sensors.

Atmospheric air pollution in the city of Krasnoyarsk is monitored by a number of federal and regional organizations (Central Siberian Department of Hydrometeorology and Environmental Monitoring, Center for Environmental Management and Environmental Protection of the Environment of the Krasnoyarsk Region, Main Department of the Ministry of Emergencies of the Krasnoyarsk Region, Siberian Federal University, etc.). Each organization possesses its own methods, technologies, and systems for collecting, storing, and processing data. Moreover, the availability of devices for estimating the air pollution level makes it possible for social ecological organizations, independent activists, and bloggers to participate in data collection too. The variety of solutions used and the interdepartmental and organizational disunity lead to the fact that a complex analysis and rapid assessment of the entire array of determined information are technically difficult at the moment and practically not carried out. With account for expansion of the number of stationary posts for observing the environmental state, which is planned by many organizations, the situation may only worsen in the coming years.

Research and development in the software and hardware for monitoring the atmospheric pollution, carried out at the Institute of Computational Modeling of the Siberian Branch of the Russian Academy of Sciences, are aim at solving the above-mentioned problem. First, a web geographical information system for monitoring the atmospheric pollution is developed. Its special feature is the capability of presenting information about the atmospheric pollution in simple visual form on the basis of the AQI. Second, a problem of collecting information about the atmospheric pollution of the city is solved, with this information received from different sources at the centralized database (DB). Within the framework of organized information communication with authorized regional subordinate organizations, information is collected from automated atmospheric observation points in real time and a web interface for analytical processing and presentation of data is developed. Third, a working prototype of the device for measuring the level of atmospheric pollution by fine dust, which provides automatic information communication on the pollution level to the DB of the monitoring system via the Global System for Mobile communications (GSM).

Suspended PM2.5 particles are one of the main pollutants of the atmosphere (data of the Department of the Federal Service for Supervision of Consumer Rights Protection and Human Well-Being (also known as Rospotrebnadzor) in the Krasnoyarsk region), but the federal and regional systems for monitoring the atmosphere in the city of Krasnoyarsk have very limited capabilities in measuring PM2.5 concentrations. The cost of systems for detecting PM2.5 suspended particle concentrations with certified sensors is rather high (in the current economic conditions, the creation of a detailed network of observations seems unlikely). At the same time, the prime cost of the proposed device is only a few thousand rubbles, and the measurement error does not exceed $10 \%$. This makes it possible to talk about a potential possibility to install tens or hundreds of devices in the city. As a result, there may be a scientific research network of observations of atmospheric pollution by PM2.5 dust. This network could allow for revealing problem regions and instances in the spatial-temporal distribution of pollution and become a good "research basis of the subsequent detailed analysis of ecological using certified devices for corresponding ecological organizations.

In general, the advantage of the proposed approach is the unique combination of free software based web GIS, constructed in the service-oriented architecture of the geoportal, and the IT-infrastructure of data collection, providing integration of operational information of various urban systems for the atmosphere monitoring. Using a relatively cheap device to measure the dust concentration can contribute significantly to solving environmental problems.

The purpose of this proposed work is to review the possibilities of the developed software and hardware for monitoring the atmospheric pollution, computational technologies, and data analysis methods.

\section{ASSESSING AIR POLLUTION ON THE BASIS OF THE AIR QUALITY INDEX}

The quality of atmospheric air is the most important environmental factor, which determines the health of the population and the state of ecosystems. For the purpose of integrated assessment of the degree of air pollution, most countries of the world use unified indicators, such as air quality index (AQI) [9], which is used to send information on the air pollution to the public in a simple and visual form. The main idea is that each substance has a scale of pollution levels, consisting of several classes as a function of the degree of influence on human health. The quality index is calculated on the basis of concentration indices of several

OPTOELECTRONICS, INSTRUMENTATION AND DATA PROCESSING Vol. 54 No. 32018 
pollutants: suspended particles with a diameter less than $10 \mu \mathrm{m}$ (PM10) and smaller than $2.5 \mu \mathrm{m}$ (PM2.5), carbon dioxide $(\mathrm{CO})$, sulfur dioxide $\left(\mathrm{SO}_{2}\right)$, nitrogen dioxide $\left(\mathrm{NO}_{2}\right)$, and ozone $\left(\mathrm{O}_{3}\right)$. For each class of the scale of pollution levels, a color designation is introduced (green/yellow/red/burgundy/black colors stand for the corresponding degree of air pollution and influence on human health, from a safe level to a natural disaster), and recommendations to the population are formulated.

The peculiarity and advantage of the web GIS considered in this paper is its capability to present the pollution data in the form of both AQIs and its modifications (InstantCast, NowCast, NowCastChina, etc.), the use of which greatly facilitates the perception of information by ordinary non-specialist users.

\section{SYSTEM FOR COLLECTING, PRESENTING, AND ANALYZING OPERATIONAL OBSERVATION DATA}

The source of information on the atmospheric pollution level is the Departmental Information and Analytical System on the State of the Environment of the Krasnoyarsk region, with the information being collected since 2009. The atmospheric air quality is observed at six automated observation stations in the city of Krasnoyarsk, at which the equipment is located that provides continuous automatic measurement of mass concentrations of oxide and nitrogen dioxide, sulfur dioxide, carbon monoxide, dust, and formaldehyde in the atmospheric air. The system collects, processes, stores, and transfers the collected information to a remote server. The concentrations of pollutants in the air are measured along with meteorological parameters (wind direction and velocity, temperature, humidity, and atmospheric pressure). Moreover, in certain neighborhoods of the city of Krasnoyarsk, the air pollution (ammonia, hydrogen sulfide, hydrochloride, hydrofluoride, benzapyrene and suspended substances) is observed everyday with the use of a mobile laboratory (on a sliding schedule).

The information collection system is implemented on the basis of software and hardware of the geoportal of the ICM SB RAS [10-12]. The observation results is accessed by standard means of the geoportal, which includes viewing the tabular data, exporting, and viewing the data on maps with the ability to choose the time intervals. A distinctive feature is the flexible configuration of visualization and web services of import/export of all available information.

The observation results can be view with the help of the existing cartographic web interface of the geoportal [13]. The main elements of the interface are a base map and observation data in form of a semitransparent layer at a given time for a certain index. Users can work with such control elements as the choice of the certain index and a time interval. Additional instruments can be used to view data with a certain time step in one of the directions. Anomalies can be sought for by displaying data in form of an active graph showing maximum values with a quick transition to viewing them at a certain instance (Fig. 1).

The information is uploaded to the geoportal by means of special software with a corresponding driver for processing and converting the input data and for periodical uploading of given observations via a web service in a JSON format, which contains three sections of information: observation posts and their coordinates, a list of indices (pollutants and meteorological data), and time based values of indices $[14,15]$.

The load on the remote server during uploading of the archive of the 2009-2017 observation data is minimized by creating text files in a JSON format. The import of data direct from the DB on the remove server to the geoportal server takes several days. After the archive is imported, the data are uploaded once an hour, and the data of the mobile laboratories are uploaded once every few days. If there is a problem in data import from a remote web service, the developed software automatically finds the date of the recent data obtained and forms a request to upload new ones. The load on the remote service is minimized by uploading the information in portions with a certain time interval until the current time is not achieved in the data.

\section{DEVICE FOR MEASURING THE ATMOSPHERIC POLLUTION LEVEL}

The device for detecting the concentration of fine suspended particles (PM2.5) with a size of $2.5 \mu \mathrm{m}$ is designed and created on the basis of electronic components available on the market. The system is based on an Arduino microcontroller, which is an electronic designer and user-friendly platform for the rapid development of devices, which is very popular all over the world thanks to convenience and simplicity of the programming language, as well as open architecture and software code. The microcontroller is programmed using the Wiring language (a simplified version of $\mathrm{C}++$ ) and Arduino IDE development framework.

OPTOELECTRONICS, INSTRUMENTATION AND DATA PROCESSING Vol. 54 No. 32018 


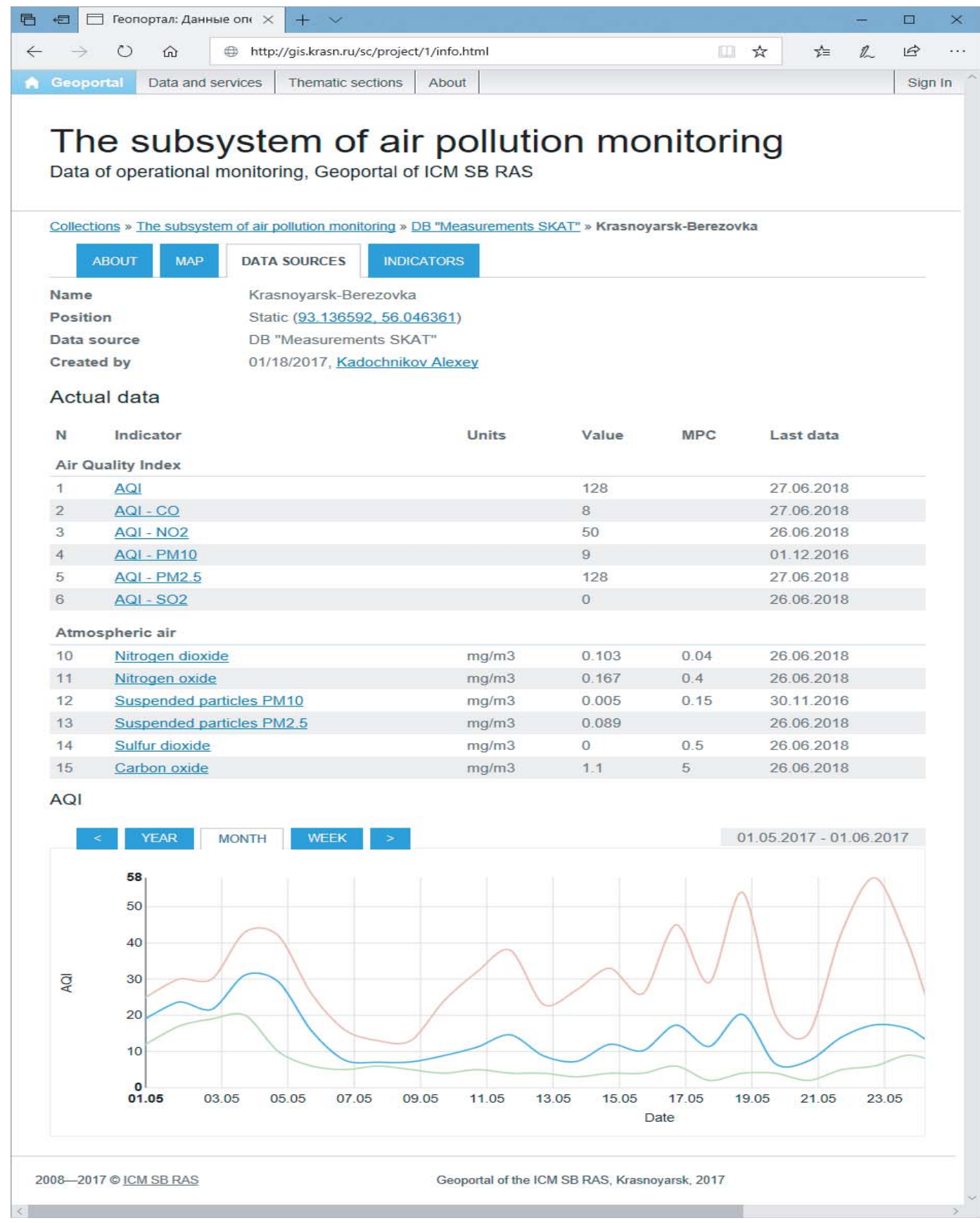

Fig. 1. Rapid assessment data at the geoportal of the ICM SB RAS (the graph shows the variation dynamics of the AQI - mean values, minima, and maxima for the selected time period (May 2017)). 


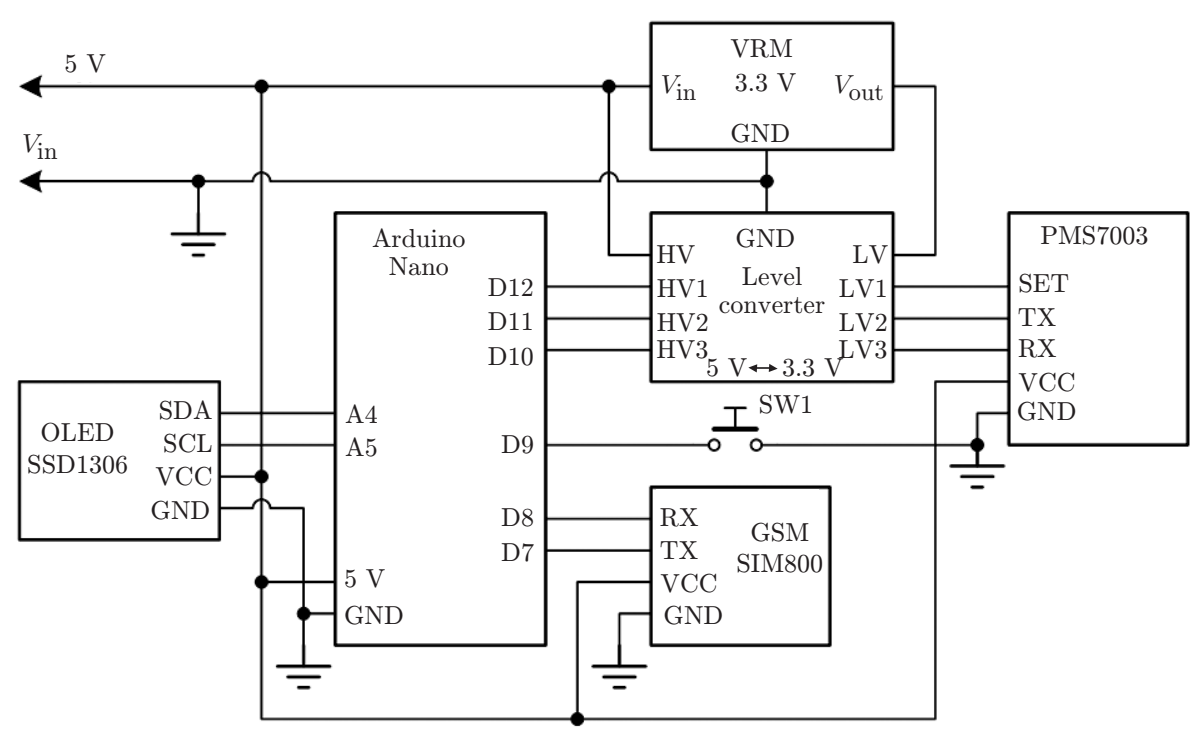

Fig. 2. Principle diagram of the device for measuring the atmospheric pollution level.

The principle diagram of the device and its main components are illustrated in Fig. 2:

(1) Arduino Nano 3.0 is a microcontroller with strapping, from the series of various modifications of which the board is chosen based on a sufficient memory capacity, the number of input-output ports, miniature sizes, and user friendliness;

(2) PMS7003 is the sensor of the suspended particles;

(3) SIM800 is the GSM/GPRS module for data transmission to a server, in which a TCP/IP protocol stack is implemented and HTTP requests can be executed;

(4) OLED is a $128 \times 64$ monochrome matrix display on a SSD1306 controller;

(5) VRM $3.3 \mathrm{~V}$ is a linear stabilizer, which reduces the supply voltage to $3.3 \mathrm{~V}$;

(6) LevelConverter is a logic level converter for matching modules with different voltages of logic levels (Arduino Nano and PMS7003).

The measuring element of the developed device for determining the concentration of suspended particles is a PMS7003 module, which a universal digital sensor made by Plantower [16]. The sensor is based on measuring the scattering of laser radiation on suspended particles. The sensor has a digital serial interface through which the measured values of the dust concentration are transmitted to the Arduino macrocontroller.

For the microcontroller, an internal microprogram is developed using the Wiring language. There are several modes of operation of the device with different periods of display and data transfer to the server (time of the screen update / data transfer): $5 \mathrm{~s} / \mathrm{off}, 5 \mathrm{~s} / 20 \mathrm{~s}, 5 \mathrm{~s} / 20 \mathrm{~m}$, and $20 \mathrm{~m} / 20 \mathrm{~m}$. In modes with a long period between measurements, there is a temporary shutdown of the internal dust sensor fan for saving its operational resource. The application is constructed on the basis of a task scheduler, which makes it possible to unload the main program cycle and make logic in the form of separate tasks with a controlled launch period.

A number of libraries with an open source code are used for programming the device: Agenda - for the implementation of the task scheduler, SSD1306Ascii - for working with an OLED-display on the SSD1306 controller, and TinyGSM - for data transfer via the cellular network. The PMS7003 sensor appeared on the market quite recently, and there are no convenient libraries yet to work with it on an Arduino framework. The Martin Falatic programming code is the basis for its library for receiving data from the PMS7003 sensor.

At the stage of preliminary research of the capabilities of selected components, the device is assembled and debugged on a breadboard. Later, the prototype is assembled in a $100 \times 60 \times 25$-mm compact package. The device needs an external power source connected via a standard microUSB connector (Fig. 3).

\section{MONITORING SYSTEM PROGRAMMING INTERFACE}

The data on the air pollution by suspended particles is collected and processed via the SensorCollector service of the geoportal of the ICM SB RAS. The program interface of the service is constructed on the

OPTOELECTRONICS, INSTRUMENTATION AND DATA PROCESSING Vol. 54 No. 32018 

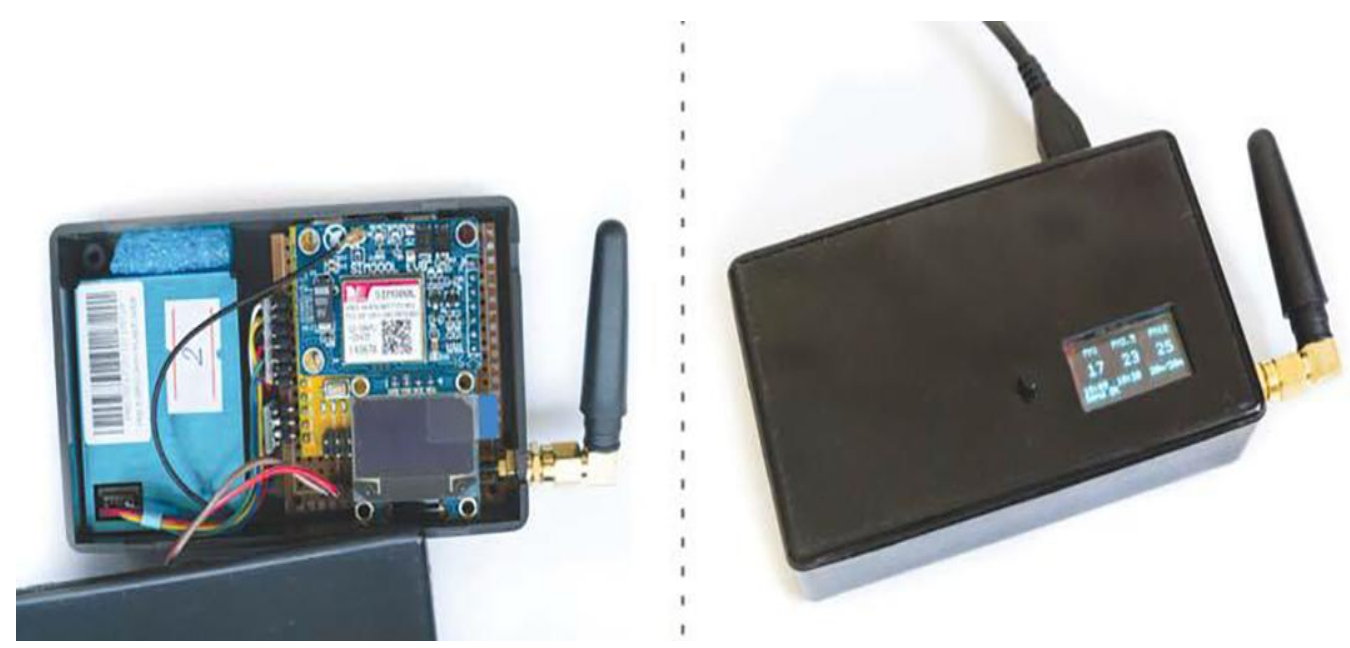

Fig. 3. Prototype of the device for measuring the atmospheric pollution level.

basis of the REST approach, the requests are transmitted by the HTTP methods of GET/POST/DELETE. The current values of the indices are transmitted by the following query:

http://gis.krasn.ru/sc/api/1.0/projects/〈project_id $\rangle /$ values/send?key $=\langle$ key $\rangle \&$ site $=\langle$ site_id $\rangle \&\langle$ values $\rangle$,

where $\langle$ key $\rangle$ is the user key for accessing the service; $\langle$ project_id $\rangle$ is the the project identifier in which the data are collected, 〈site_id $\rangle$ is the site identifier on which the sensor is located, and 〈values $\rangle$ stands for the index values transmitted by individual parameters.

\section{WEB INTERFACE OF THE AIR POLLUTION MONITORING SYSTEM}

A user interface for the web GIS for monitoring is designed and developed on the basis of program interfaces of the geoportal of the ICM SB RAS, a set of previously developed hardware and software [17]. The web application forms are designed by using the mechanism of formation of dynamic content on the basis of templates. The templates can change the order and form of the display of attribute data on the objects in the map layers, including various elements of the style design: color, font parameters, etc.

\section{CONCLUSION}

A web-based geoinformation analytical system for monitoring the atmospheric pollution is developed on the basis of technologies of the geoportal of the ICM SB RAS. The problem of automatic collection of information on the level of atmospheric pollution in the city coming from different sources into a centralized database is solved. The display of information on the level of atmospheric pollution in a simple visual form on the basis of the air quality index is implemented. The available components are used to develop a working prototype of the device to measure the atmospheric pollution by fine dust and automatically transfer the data to the monitoring system. The device is in trial operation, with the current version being able to transfer the values of PM1, PM2.5, and PM10 over the cellular network. Further, the list of indicators should be expanded by adding the total number of suspended particles per unit volume, temperature, humidity, and atmospheric pressure.

\section{REFERENCES}

1. A. G. Ashitko and I. V. Man'shina, "System for Monitoring the State of Atmospheric Air in the City of Kaluga," Vestn. Kaluzh. Univ. 22 (1), 5-9 (2014).

2. CityAir. City Quality Monitoring. http://cityair.ru.

3. D. T. Azyomov, "System for Monitoring Atmospheric Air of St. Petersburg," Okruzh. Sreda Sankt-Peterburga 2 (2), 8-14 (2016). 
4. V. S. Karpov, V. M. Panarin, A. A. Goryunkova, "Hardware-Software System for Estimating the Atmospheric Air Pollution," Izv. Tul. Gos. Univ. Tekhnicheskie Nauki 2, 173-182 (2012).

5. S. Wiemann, J. Brauner, P. Karrasch, et al., "Design and Prototype of an Interoperable Online Air Quality Information System," Environmental Modeling \& Software 79, 354-366 (2016).

6. T. Savu, B. A. Jugravu, and D. Dunea, "On the Development of a PM2.5 Monitoring Network for Real-Time Measurements in Urban Environments," Revista de Chimie 68 (4), 796-801 (2017).

7. L. Hu, P. Yue, M. Zhang, et al., "Task-Oriented Sensor Web Data Processing for Environmental Monitoring," Earth Sci. Inform. 8 (3), 511-525 (2015).

8. A. Zaldei, F. Camilli, T. de Filippis, et al., "An Integrated Low-Cost Road Traffic and Air Pollution Monitoring Platform for Next Citizen Observatories," Trans. Research Procedia 24, 531-538 (2017).

9. Beijing Air Pollution: Real-time Air Quality Index (AQI). http://aqicn.org.

10. Geoportal of the Institute of Computational Modeling of the Siberian Branch of the Russian Academy of Sciences. http://gis.krasn.ru.

11. O. Yakubailik, A. Kadochnikov, and A. Tokarev, "GIS Development," in Proc. of the 15th Intern. Multidisciplinary Scientific GeoConf. Albena, Bulgaria, June 18-24, 2015. Book 2, Vol. 1.

12. A. G. Matveev and O. E. Yakubailik, "Designing and Developing Software and Hardware for Geospatial Web Applications," Fundamental'nye Issledovaniya 15 (10), 3358-3362 (2013).

13. A. A. Kadochnikov and O. E. Yakubailik, "Development of Software for Collecting and Visualizing the Observational Data for the Geoportal of the Institute of Computational Modeling of the Siberian Branch of the Russian Academy of Sciences," Vestn. Nov. Gos. Univ. Informatsionnye Tekhnologii 12 (4), 23-31 (2014).

14. A. A. Kadochnikov, "Construction of Geospatial Web Applications and Services for Environmental Monitoring," Vestn. Nov. Gos. Univ. Informatsionnye Tekhnologii 12 (4), 23-31 (2014).

15. O. E. Yakubailik, "Technologies for the Formation of Interactive Thematic Maps on the Geoportal," Vestn. Komp. Informatsionnykh Tekhnologii 154 (4), 23-28 (2017).

16. The Plantower PMS5003 and PMS7003 Air Quality Sensor Experiment. http://aqicn.org/sensor/pms5003-7003.

17. A. K. Matuzko and O. E. Yakubailik, "Development of Applied GIS Based on the Geoportal Technologies," Obrazovat. Resursy i Tekhnologii 14 (2), 202-209 (2016). 